\title{
Dual Billiards, Fagnano Orbits, and Regular Polygons.
}

\author{
Serge Troubetzkoy
}

In this article we consider the dual version of two results on polygonal billiards. We begin by describing these original results. The first result is about the dynamics of the so-called pedal map related to billiards in a triangle $P$. The three altitudes of $P$ intersect the opposite sides (or their extensions) in three points called the feet. These three points form the vertices of a new triangle $Q$ called the pedal triangle of the triangle $P$ (Figure 1). It is well known that for acute triangles the pedal triangle forms a period-three billiard orbit often referred to as the Fagnano orbit, i.e., the polygon $Q$ is inscribed in $P$ and satisfies the usual law of geometric optics (the angle of incidence equals the angle of reflection) or equivalently (this is a theorem) the pedal triangle has least perimeter among all inscribed triangles. The name Fagnano is used since in 1775 J. F. F. Fagnano gave the first proof of the variational characterization. In a sequence of elegant and entertaining articles, J. Kingston and J. Synge [5, P. Lax [6], P. Ungar [11, and J. Alexander [1] studied the dynamics of the pedal map given by iterating this process. The second result, due to DeTemple and Robertson [3], is that a closed convex polygon $P$ is regular if and only if $P$ contains a periodic billiard path $Q$ similar to $P$.

There is a dual notion to billiards, called dual or outer billiards. The game of dual billiards is played outside the billiard table. Suppose the table is a polygon $P$ and that $z$ is a point outside $P$ and not on the continuation of any of $P$ 's

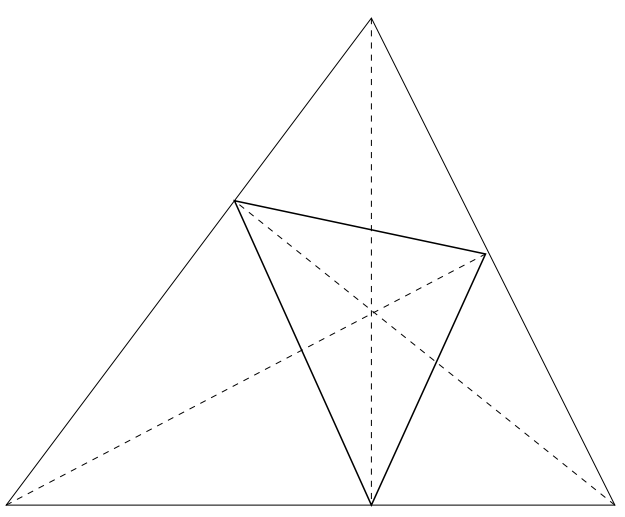

Figure 1: A pedal triangle. 


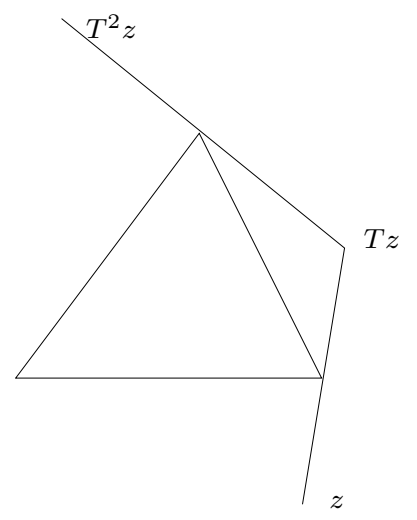

Figure 2: The polygonal dual billiard map.

sides. A line $L$ is a support line of $P$ if it intersects the boundary $\partial P$ of $P$ and $P$ lies entirely in one of the two regions into which the line $L$ divides the plane. There are two support lines to $P$ through $z$; choose the right one as viewed from $z$. If $z$ is not on the continuation of a side of the convex hull of $P$ then this support line intersects $P$ at a single point which we call the support vertex of $z$. Reflect $z$ in its support vertex to obtain $z$ 's image under the dual billiard map denoted by $T$ (see Figure 2). The map $T^{n}$ is defined for all $n$ at the point $z$ if none of its images belongs to the continuation of a side of the convex hull of the polygon. Dual billiards have been extensively studied by S. Tabachnikov (see [7- 10] and the reference therein).

Throughout the article we will identify the polygon having vertices $z_{i} \in \mathbb{C}$, $i=1, \ldots, n$, ordered cyclically with the point $z=\left(z_{1}, \ldots, z_{n}\right) \in \mathbb{C}^{n}$. In particular a polygon for us is an oriented object. The notion of polygon includes self-intersecting polygons (which we call star shaped polygons) and geometrically degenerate $n$-gons: those with a side of length 0 , or an angle of $\pi$ or $2 \pi$ at a vertex. Corresponding to the fact that the Fagnano billiard orbit hits each side of the triangle, we call an $n$-periodic dual billiard orbit consisting of $n$ points consecutively reflected in the vertices $z_{1}, z_{2}, \ldots, z_{n}$ a Fagnano dual billiard orbit. Fagnano orbits for dual billiards were introduce by Tabachnikov in [8], where he studied a certain variational property analogous to one in the pedal case studied by Gutkin [4].

Motivated by the notion of pedal triangles, we introduce here dedal $n$-gons. An $n$-gon $Q$ is called a dedal $n$-gon of the $n$-gon $P$ if reflecting the vertex $w_{i}$ of $Q$ in the vertex $z_{i}$ of $P$ yields the vertex $w_{i+1}$. (Throughout the article all subscripts will be taken modulo $n$ without explicit mention.) There is no requirement that the sides of $Q$ touch $P$ only at a vertex. From the definition it is clear that if a Fagnano orbit of an $n$-gon $P$ exists then it is a dedal $n$-gon. A nondegenerate polygon can have a degenerate dedal polygon and vice-versa. Examples are shown in Figures 3 and 4 . The degeneracy not shown cannot occur: i.e., it is impossible for two consecutive vertices of $P$ to coincide if $Q$ is 

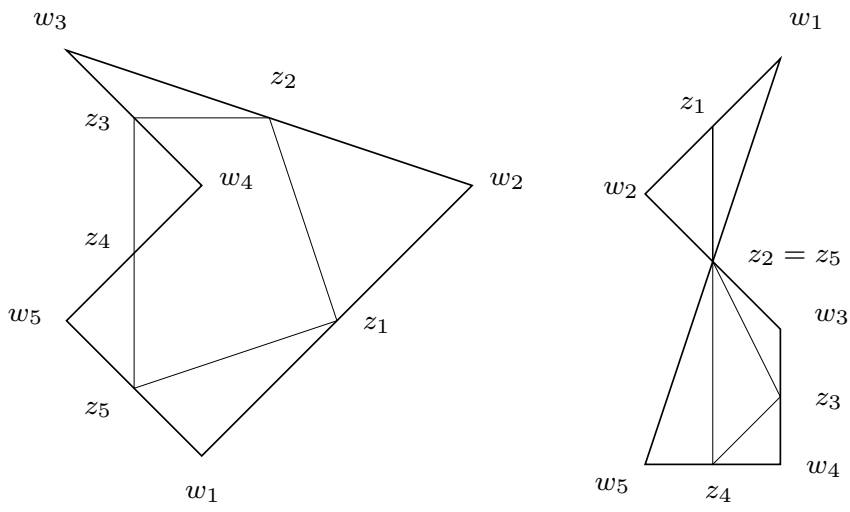

Figure 3: Nondegenerate dedal pentagons corresponding to degenerate pentagons, with angle $\pi$ and $2 \pi$.
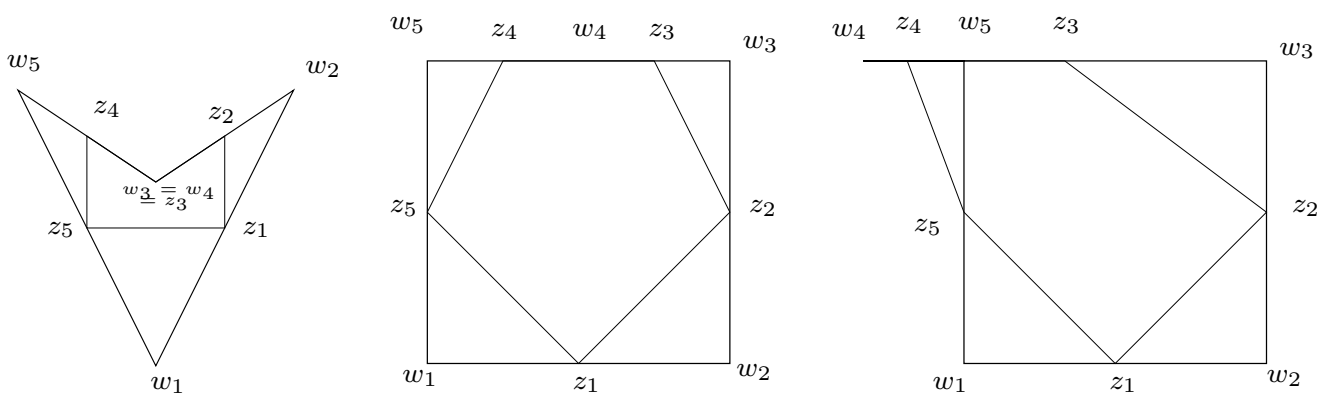

Figure 4: Degenerate dedal polygons: loss of a vertex, angle $\pi$, and angle $2 \pi$.

nondegenerate. We will not dwell on this aspect.

In this article we study the dedal $n$-gon(s) $Q$ of an $n$-gon $P$. Our main results are the following. If $n$ is odd then its dedal $n$-gon exists and is unique. For $n$ even we give a necessary and sufficient condition for the existence of dedal $n$-gons and describe the space of all dedal $n$-gons of $P$. Then we go on to characterize regular and affinely regular $n$-gons by similarity to their dedal $n$-gons. Finally we give a complete description of the dynamics of the developing map $\mu(Q):=P$. The proofs of all our results boil down to some linear algebra of the dedal map.

After we wrote this article one of the anonymous referees pointed out that iteration of the developing map has already been studied by Berlekamp, Gilbert, and Sinden in 1965 [2]. They answer a question they attribute to G. R. MacLane, namely, they prove that for almost every polygon $Q$ there exists an $M \geq 1$ such that $\mu^{M}(Q)$ is convex. Note that the image of a convex polygon is convex; thus this implies that for almost every $Q$ there exists an $M$ such that $\mu^{m}(Q)$ is convex for all $m \geq M$. 
Suppose $Q\left(w_{1}, \ldots, w_{n}\right)$ is a dedal polygon of $P\left(z_{1}, \ldots, z_{n}\right)$. The definition implies that

$$
z_{i}=\left(w_{i}+w_{i+1}\right) / 2
$$

(see Figures 3, 4, and 5). The linear transformation $\mu: \mathbb{C}^{n} \rightarrow \mathbb{C}^{n}$ given by $\mu\left(w_{1}, \ldots, w_{n}\right)=\left(z_{1}, \ldots, z_{n}\right)$ is called the developing map.

The characteristic polynomial of $\mu$ is

$$
(1-2 x)^{n}-(-1)^{n} .
$$

Its eigenvalues are $\left(1+q^{i}\right) / 2$ for $i=0,1, \ldots, n-1$, where $q:=\exp (2 \pi i / n)$. All the eigenvalues differ from zero except for the $(n / 2)$ th eigenvalue when $n$ is even. The $i$ th eigenvector is $X_{i}:=\left(1, q^{i}, q^{2 i}, \ldots, q^{(n-1) i}\right)$. The vectors $X_{i}$ form a basis of our space of polygons. If $i$ divides $n$ then $X_{i}$ is a polygon with $n / i$ sides. Nonetheless for the sake of clarity (avoiding stating special cases) we will think of this as an $n$-gon which is traced $i$ times; for example if $n=6$ then $X_{2}=\left(1, q^{2}, q^{4}, q^{6}, q^{8}, q^{10}\right)=\left(1, q^{2}, q^{4}, 1, q^{2}, q^{4}\right)$ traces the triangle $\left(1, q^{2}, q^{4}\right)$ twice. An exception to this rule is the case $n$ even and $i=n / 2$. In this case $X_{n / 2}$ is a segment which we do not consider as a polygon.

The following proposition clarifies the existence of dedal polygons.

Proposition 1 a) Suppose that $n \geq 3$ is odd. Then for any $n$-gon $P$ there is a unique dedal n-gon $Q$.

b) If $n \geq 3$ is even then dedal $n$-gons exist if and only if the vertices of $P$ satisfy $z_{1}-z_{2}+z_{3}-\cdots-z_{n}=0$. If this equation is satisfied then there is a unique dedal n-gon $Q_{0}$ in the space $X_{n / 2}^{\perp}:=\left\{z \in \mathbb{C}^{n}: z \cdot X_{n / 2}=0\right\}$. The set $D:=\left\{Q_{0}+s X_{n / 2}: s \in \mathbb{C}\right\}$ consists of the dedal n-gons of $P$. In particular for each $i \in\{1, \ldots, n\}$ every point $w \in \mathbb{C}$ is the ith vertex of a unique dedal $n$-gon $Q_{i}(w)$.

We remark that the condition $z_{1}-z_{2}+z_{3}-\cdots-z_{n}=0$ means that the center of mass of the even vertices coincides with the center of mass of the odd vertices. Proof of Proposition 1. If $n \geq 3$ is odd then the map $\mu$ is invertible with

$$
w_{i}=z_{i}-z_{i+1}+z_{i+2}-\cdots+z_{i-1},
$$

and thus the dedal polygon exists and is unique.

On the other hand, if $n \geq 3$ is even then the map $\mu$ is not invertible. The kernel is one (complex) dimensional and is generated by the vector $X_{n / 2}:=$ $(1,-1,1,-1, \ldots, 1,-1)$. Dedal polygons exist if and only if $z=\left(z_{1}, z_{2}, \ldots, z_{n}\right)$ is in the range of $\mu$, i.e., the space spanned by the $X_{i}$ for $i \neq n / 2$. The range and kernel of $\mu$ are orthogonal since if $i \neq n / 2$ then

$X_{i} \cdot X_{n / 2}=\left(1+q^{2 i}+q^{4 i}+\cdots+q^{(n-2) i}\right)-\left(q^{i}+q^{3 i}+\cdots+q^{(n-1) i}\right)=0-0=0$.

Thus $z$ is in the range of $\mu$ if and only if it satisfies $z \cdot X_{n / 2}=0$, or equivalently

$$
z_{1}-z_{2}+z_{3}-\cdots-z_{n}=0 .
$$




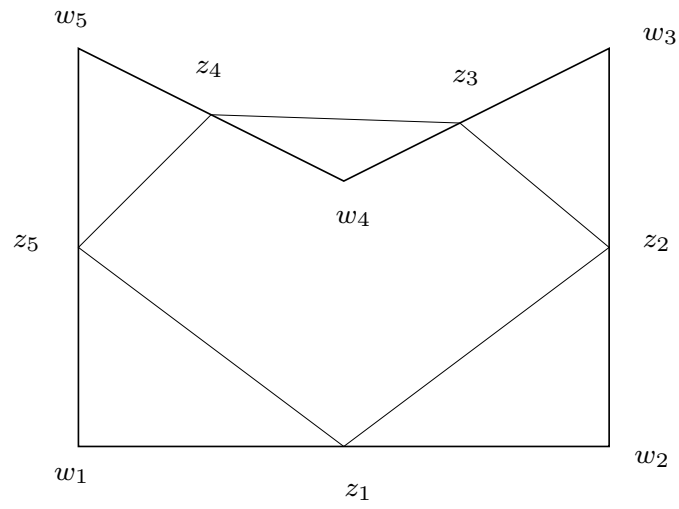

Figure 5: A pentagon without a Fagnano orbit. The unique dedal pentagon is pictured.

Alternatively, to see this note that

$$
\begin{aligned}
& z_{1}+z_{3}+\cdots+z_{n-1}=\frac{1}{2}\left(\left(w_{1}+w_{2}\right)+\left(w_{3}+w_{4}\right)+\cdots+\left(w_{n-1}+w_{n}\right)\right) \\
& \quad=\frac{1}{2}\left(\left(w_{2}+w_{3}\right)+\left(w_{4}+w_{5}\right)+\cdots+\left(w_{n}+w_{1}\right)\right)=z_{2}+z_{4}+\cdots+z_{n} .
\end{aligned}
$$

The uniqueness of $Q_{0}$ follows since the map $\mu$ is invertible on the space $X_{n / 2}^{\perp}$. The statement about the set $D$ follows immediately since $X_{n / 2}$ is the kernel of $\mu$. Let $Q_{0}:=\left(w_{1}^{0}, \ldots, w_{n}^{0}\right)$. For each $i$, any point $w \in \mathbb{C}$ can be uniquely expressed as $w_{i}^{0}+s$ for some $s \in \mathbb{C}$.

Suppose that a polygon $Q$ without self intersection is a dedal polygon of $P$. If $Q$ is convex then it is clearly a Fagnano orbit of $P$ since by convexity the polygon $P$ must be contained in $Q$. On the other hand if $Q$ is not convex then it cannot be a Fagnano orbit since $P$ cannot be contained in $Q$. In particular Fagnano orbits always exist for triangles, but not for polygons with more sides. An example of a pentagon without a Fagnano orbit is given in Figure 5. Although not every polygon has a Fagnano orbit, it does have a periodic orbit that is nearly as simple. Namely, consider the second iteration $T^{2}$ of the dual billiard map. Connecting the consecutive points of a periodic orbit of $T^{2}$ yields a polygon. Cutler has shown that the map $T^{2}$ has a periodic orbit which lies outside any compact neighborhood of $P$ and the polygon constructed from the orbit makes a single turn about $P[9]$. The existence of a periodic orbit for the usual billiard in an arbitrary polygon remains open.

The set of polygons with center of mass at the origin is

$$
\mathrm{C}:=\left\{\left(w_{1}, \ldots, w_{n}\right) \in \mathbb{C}^{n}: w_{1}+\cdots+w_{n}=0\right\} .
$$

The eigenvector $X_{0}=(1,1, \ldots, 1)$ represents a polygon which degenerates to a point. The set $\mathrm{C}$ is the orthogonal complement of $X_{0}$, i.e., $\mathbf{C}=\{w=$ $\left.\left(w_{1}, \ldots, w_{n}\right) \in \mathbb{C}^{n}: w \cdot X_{0}=0\right\}$. We will express polygons in the eigenbasis, i.e., we write $P=\sum_{i=0}^{n-1} a_{i} X_{i}$; the coefficients $a_{i}$ are complex numbers. 


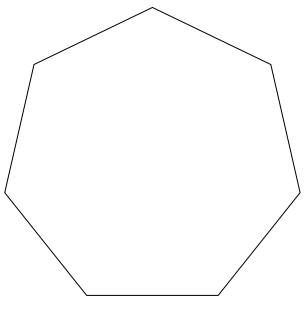

(a)

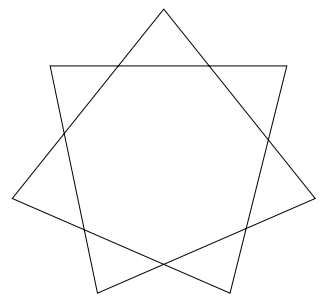

(b)

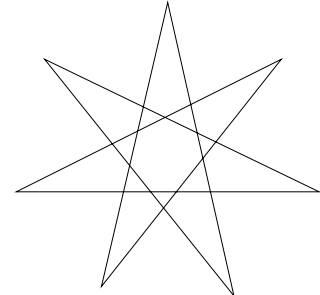

$(c)$

Figure 6: Up to orientation there are three regular 7-gons.

Since the map $\mu$ preserves the center of mass, throughout the rest of the article we assume that the center of mass is at the origin, i.e., $a_{0}=0$.

$X_{1}$ and $X_{n-1}$ are the usual regular $n$-gon in counterclockwise and clockwise orientation (Figure 6a). If $i$ and $n$ are relatively prime and $i \notin\{1, n-1\}$ then $X_{i}$ is star shaped and we also call it regular (Figures $6 \mathrm{~b}$ and c). Finally if $i$ divides $n$ and $i \neq n / 2$ then $X_{i}$ is naturally a regular $n / i$-gon which, as mentioned above, we will think of as (a multiple cover of) a regular $n$-gon.

Two (unoriented) polygons are called similar if all corresponding angles are equal and all distances are increased (or decreased) in the same ratio. Since we study oriented ordered polygons we additionally want the marking of the vertices of $P$ and $Q$ to correspond, in which case we will call $P$ and $Q \star$-similar. Thus two polygons $P=\sum_{i=1}^{n-1} b_{i} X_{i}$ and $Q=\sum_{i=1}^{n-1} a_{i} X_{i}$ are $\star$-similar if there exists a nonzero complex constant $\ell$ such that $b_{i}=\ell a_{i}$ for all $i$. We will also write this as $P=\ell Q$. Note that if $P$ and $Q$ are $\star$-similar then they are similar. On the other hand if $P$ and $Q$ are similar then $P$ is $\star$-similar to a cyclic permutation $Q^{(k)}:=\left(w_{k}, w_{k+1}, w_{k+2}, \ldots, w_{k-1}\right)$ of $Q$ or a cyclic permutation $\bar{Q}^{(k)}:=\left(w_{k}, w_{k-1}, w_{k-2}, \ldots, w_{k+1}\right)$ of $Q$ with the opposite orientation.

In analogy to DeTemple and Robertson's result we have:

Theorem 2 Fix $n \geq 3$. An n-gon $P$ is regular if and only if it has a dedal polygon $Q$ which is $\star$-similar to $P$.

Note that if $n$ is odd then $Q$ is the unique dedal polygon of $P$, while if $n$ is even then $Q$ is the unique dedal polygon $Q_{0} \in X_{n / 2}^{\perp}$.

Proof. Suppose $P$ is regular, i.e., there is a nonzero complex constant $\ell$ such that $P=\ell X_{j}$, where $j \neq n / 2$ if $n$ is even. If $n$ is odd then let $Q$ be the unique dedal polygon of $P$. If $n$ is even then since $P$ is regular it satisfies (11). Thus $P$ has dedal polygons and we choose $Q=Q_{0} \in X_{n / 2}^{\perp}$. In both cases let $Q=\sum a_{i} X_{i}$. Since $P=\mu(Q)=\sum \frac{1+q^{i}}{2} a_{i} X_{i}$, we have $a_{i}=0$ for $i \neq j$, i.e., $Q=a_{j} X_{j}$. Thus $Q$ is $\star$-similar to $P=\ell X_{j}$.

Conversely suppose that $Q=\sum a_{i} X_{i}$ is a dedal polygon of $P=\sum b_{i} X_{i}$ and that $P$ and $Q$ are $\star$-similar, i.e., there is a nonzero complex constant $\ell$ such that $a_{i}=\ell b_{i}$. Since $P=\mu(Q)$ we have $\frac{1+q^{i}}{2} a_{i}=b_{i}$ for $i=1, \ldots, n$. Combining this 
with the $\star$-similarity yields $\frac{1+q^{i}}{2}=1 / \ell$ (a constant) for each $i$ such that $a_{i} \neq 0$. It follows $q^{i}=q^{j}$ if $a_{i} \neq 0$ and $a_{j} \neq 0$. Since $q^{i} \neq q^{j}$ for $i \neq j$ it follows that only a single $a_{j}$ is nonnull. Therefore $Q=a_{j} X_{j}$ and thus $P=\ell^{-1} a_{j} X_{j}$, and both are regular. Note that if $n$ is even then $i \neq n / 2$, since if $i=n / 2$ then $\left(1+q^{i}\right) / 2=0$ and thus $b_{i}=a_{i}=0$.

Of course we would like to have the analog of Theorem 2 with the usual notion of similarity. We call an $n$-gon affinely regular if there exists a $j(j \neq$ $n / 2$ when $n$ is even) such that $P$ is in the subspace $A_{j}$ generated by $X_{j}$ and $X_{n-j}$. This is equivalent to $P$ being the image of a regular $n$-gon by an affine transformation, which explains the name.

Theorem 3 a) Suppose $n \geq 3$ is odd. An n-gon $P$ is affinely regular if and only if it has a dedal polygon $Q$ which is similar to $P$.

b) If $n \geq 4$ is even then an $n$-gon $P$ appears in the following list of affinely regular polygons if and only if it has a dedal polygon $Q$ which is similar to $P$.

i) Regular n-gons

ii) Any $P \in A_{j}$ such that there exists $k \in\{1, \ldots, n\} \backslash\{n / 2\}$ such that $n$ divides $j(2 k-1)$

iii) Any $P=b_{j} X_{j}+b_{n-j} X_{n-j} \in A_{j}$ such that there exists $k \in$ $\{1, \ldots, n\}$ with $b_{j} / b_{n-j}= \pm q^{j(k+3 / 2)}$

All triangles are affinely regular. Berlekamp et al. noticed that every triangle is similar to its dedal triangle [2]. One would like to know if there are other special properties of the polygons in the list.

Proof. Suppose $Q^{(1)}=Q=\sum_{i=1}^{n-1} a_{i} X_{i}$. Then it is a simple exercise in linear algebra to verify that $Q^{(k)}=\sum a_{i} q^{i(k-1)} X_{i}$ and that $\bar{Q}^{(k)}=\sum a_{n-i} q^{i(k+1)} X_{i}$. Thus the similarity of $P$ to $Q$ is equivalent to the existence of a $k \in\{1, \ldots, n\}$ and a nonzero complex constant $\ell$ such that $b_{i}=\ell a_{i} q^{i(k-1)}$ for $i=1, \ldots, n-1$ or $b_{i}=\ell a_{n-i} q^{i(k+1)}$ for $i=1, \ldots, n-1$.

The structure of the proof is as follows. We group the even and odd cases together and start by proving that the various classes of affinely regular polygons are similar to their dedal polygons. Then we turn to the converse.

Suppose that $n$ is odd and $P=b_{j} X_{j}+b_{n-j} X_{n-j}$ is affinely regular. Let $Q=Q^{(1)}=\sum a_{i} X_{i}$ be the unique dedal polygon of $P$. Since $P=\mu(Q)=$ $\sum \frac{1+q^{i}}{2} a_{i} X_{i}$, we have $a_{i}=0$ for $i \notin\{j, n-j\}$. We claim that there is a nonzero complex constant $\ell$ such that $P=\ell Q^{((n+3) / 2)}$. To see this note that

$$
1+q^{j}=q^{j}\left(1+q^{-j}\right)=q^{j(n+1)}\left(1+q^{-j}\right)=q^{j(n+1) / 2} q^{j(n+1) / 2}\left(1+q^{-j}\right)
$$

or

$$
\frac{1+q^{j}}{2 q^{j(n+1) / 2}}=\frac{1+q^{-j}}{2 q^{-j(n+1) / 2}} .
$$

Thus $P=\ell Q^{((n+3) / 2)}$ for $\ell:=\left(1+q^{j}\right) /\left(2 q^{j(n+1) / 2}\right)$. 
The case $n$ is even and $P$ of class (ii) is similar. Consider the dedal polygon $Q=Q_{0} \in X_{n / 2}^{\perp}$. Again $P=\mu(Q)$ implies that $a_{i}=0$ if $i \notin\{j, n-j\}$. We claim that there is a nonzero complex constant $\ell$ such that $P=\ell Q^{(k+1)}$. Since $n$ divides $j(2 k-1)$ we have

$$
1+q^{j}=q^{j}\left(1+q^{-j}\right)=q^{j+j(2 k-1)}\left(1+q^{-j}\right)=q^{k j} q^{k j}\left(1+q^{-j}\right)
$$

or

$$
\frac{1+q^{j}}{2 q^{k j}}=\frac{1+q^{-j}}{2 q^{-k j}} .
$$

Thus $P=\ell Q^{(k+1)}$ for $\ell:=\left(1+q^{j}\right) /\left(2 q^{k j}\right)$.

The case $n$ even and $P$ regular has already been treated in Theorem 2; therefore it remains to treat case (iii) of $n$ even. We suppose $P$ is of this form and want to show that $P$ is similar to $\bar{Q}^{(k)}$, i.e., that there is a nonzero complex constant $\ell$ such that $b_{i}=\ell a_{n-i} q^{i(k+1)}$ for all $i$. As before $P=\mu(Q)$ implies that $a_{i}=0$ if $i \notin\{j, n-j\}$. Combining the two relations, $b_{i}=\frac{1+q^{i}}{2} a_{i}$ and $b_{j} / b_{n-j}= \pm q^{j(k+3 / 2)}$ yields

$$
b_{j}= \pm q^{j(k+3 / 2)} b_{n-j}= \pm q^{j(k+3 / 2)} \frac{1+q^{-j}}{2} a_{n-j}= \pm q^{j(k+1)} \frac{q^{j / 2}+q^{-j / 2}}{2} a_{n-j}
$$

and

$$
b_{n-j}= \pm q^{-j(k+3 / 2)} b_{j}= \pm q^{-j(k+3 / 2)} \frac{1+q^{j}}{2} a_{j}= \pm q^{-j(k+1)} \frac{q^{j / 2}+q^{-j / 2}}{2} a_{j} .
$$

Choosing $\ell:= \pm\left(q^{j / 2}+q^{-j / 2}\right) / 2 \in \mathbb{R}$ yields $P=\ell \bar{Q}^{(k)}$.

We turn now to the converse. We will first prove that for $n$ even or odd if $Q=\sum a_{i} X_{i}$ is similar to $P=\sum b_{i} X_{i}$ then $P$ is affinely regular.

We first treat that case when $P=\ell Q^{(k+1)}$ for some $k$ and $\ell \in \mathbb{C} \backslash\{0\}$, i.e., $b_{i}=\ell a_{i} q^{i k}$. Since $P=\mu(Q)$ we have $\frac{1+q^{i}}{2} a_{i}=b_{i}$ for $i=1, \ldots, n-1$. Note that if $n$ is even then $\left(1+q^{n / 2}\right) / 2=0$, and thus $b_{n / 2}=\frac{1+q^{n / 2}}{2} a_{n / 2}=0$ and $a_{n / 2}=\ell^{-1} q^{-n k / 2} b_{n / 2}$ is zero as well. For each $i$ such that $a_{i} \neq 0$, combining the two relations between $a_{i}$ and $b_{i}$ yields $q^{-i k} \frac{1+q^{i}}{2}=\ell$. If only a single $a_{j}$ is nonnull, then $Q$ is regular, and since $P$ is similar to $Q$ it is regular as well. Now suppose that $a_{i}$ and $a_{j}$ are nonnull. Then the previous equation implies that

$$
\left(1+q^{i}\right) /\left(1+q^{j}\right)=q^{k(i-j)} .
$$

Taking absolute values yields $\left|\left(1+q^{i}\right)\right|=\left|\left(1+q^{j}\right)\right|$, which implies $i= \pm j$. Thus $P$ is affinely regular.

If $n$ is even we need to conclude more. Note that $\left(1+q^{-j}\right) /\left(1+q^{j}\right)=q^{-j}$. Thus taking $i=-j$ in (2) implies $1=q^{j(1-2 k)}$. Thus $j(2 k-1)$ is a multiple of $n$, i.e., we are in case (ii) of the list.

Finally suppose that $P=\mu(Q)$ and $Q$ are similar but have the opposite orientation, i.e., $P=\ell \bar{Q}^{(k)}$ for some $\ell \in \mathbb{C} \backslash\{0\}$, or equivalently $b_{i}=\ell a_{n-i} q^{i(k+1)}$. 
Combing this with $\frac{1+q^{i}}{2} a_{i}=b_{i}$ yields $\frac{1+q^{i}}{2} a_{i}=\ell a_{n-i} q^{i(k+1)}$. If $n$ is even and $i=n / 2$ then this equation implies that $a_{n / 2}=0$. For all other cases it implies that $a_{i}$ and $a_{n-i}$ are simultaneous zero or nonzero. For any $i$ such that they are nonzero there are two such equations, and they imply

$$
\frac{a_{i}}{a_{n-i}}=\frac{2 \ell q^{i(k+1)}}{1+q^{i}}=\frac{1+q^{-i}}{2 \ell q^{-i(k+1)}} .
$$

This in turn yields

$$
4 \ell^{2}=\left(1+q^{i}\right) \cdot\left(1+q^{n-i}\right)=2+q^{i}+q^{n-i}:=f(i) .
$$

It is easy to see that $f(i)=f(j)$ if and only if $j=i$ or $j=n-i$. Since the left-hand side of Equation (4) does not depend on $i$, there is exactly one pair $\left(a_{j}, a_{n-j}\right)$ of nonzero coefficients, i.e., $Q$ is affinely regular. Since $P$ is similar to $Q$ it is also affinely regular.

Finally suppose that $n$ is even. Equations (3) and (14) imply that for each $j$ there exists $k \in\{1,2 \ldots, n\}$ such that

$$
\frac{b_{j}}{b_{n-j}}= \pm \frac{\sqrt{f(j)} q^{j(k+1)}}{1+q^{-j}}= \pm \sqrt{\frac{1+q^{j}}{1+q^{-j}}} q^{j(k+1)}= \pm q^{j(k+3 / 2)},
$$

i.e., we are in case (iii) of the list.

Lemma 4 If $Q$ is affinely regular then $\mu^{n}(Q)$ is $\star$-similar to $Q$.

Proof. Suppose $Q=a_{j} X_{j}+a_{n-j} X_{n-j}$. Then

$$
\mu^{n}(Q)=\left(\frac{1+q^{j}}{2}\right)^{n} a_{j} X_{j}+\left(\frac{1+q^{n-j}}{2}\right)^{n} a_{n-j} X_{n-j} .
$$

But

$$
\begin{aligned}
\left(1+q^{n-j}\right)^{n} & =\sum_{k=0}^{n}\left(\begin{array}{l}
n \\
k
\end{array}\right) q^{(n-j) k}=\sum_{k=0}^{n}\left(\begin{array}{c}
n \\
n-k
\end{array}\right) q^{-j k} \\
& =\sum_{i=0}^{n}\left(\begin{array}{c}
n \\
i
\end{array}\right) q^{-j(n-i)}=\sum_{i=0}^{n}\left(\begin{array}{c}
n \\
i
\end{array}\right) q^{j i}=\left(1+q^{j}\right)^{n} .
\end{aligned}
$$

Thus $\mu^{n}(Q)=\ell Q$ with $\ell=\left(\frac{1+q^{j}}{2}\right)^{n}$.

In analogy to the billiard results stated in the introduction we now study the dynamics of $\mu$. On the space $C$ the dynamics are not very interesting: $\mu^{m}(Q) \rightarrow$ $(0, \ldots, 0)$ as $m \rightarrow \infty$ for all $Q \in \mathrm{C}$. To get a somewhat more interesting behavior notice that $\star$-similarity is an equivalence relation. Let $[Q]:=\{\ell Q: \ell \in \mathbb{C} \backslash\{0\}\}$ denote the equivalence class of $Q$. By identifying $\star$-similar polygons we obtain 
the quotient space $\hat{\mathrm{C}}:=\{[Q]: Q \in \mathrm{C}\}$. If $Q_{1}$ and $Q_{2}$ are $\star$-similar then so are $\mu\left(Q_{1}\right)$ and $\mu\left(Q_{2}\right)$, and thus the map $\mu$ defines a map $\hat{\mu}$ of $\hat{C}$ to itself.

We remark that the equivalence class $[Q]$ of a polygon $Q=\sum a_{i} X_{i}$ can be represented by the vector $\left(a_{1}, \ldots, a_{n-1}\right) \in \mathbb{C}^{n-1} \backslash\{(0, \ldots, 0)\}$ with the identification $\left(a_{1}, \ldots, a_{n-1}\right) \equiv\left(\ell a_{1}, \ldots, \ell a_{n-1}\right)$ with $\ell \in \mathbb{C} \backslash\{0\}$. Thus the quotient space $\hat{C}$ is naturally identified with the complex projective space $\mathbb{C P}^{n-2}$.

Let $\mathrm{S}$ be the unit sphere in C, i.e., the set of all $Q=\sum a_{i} X_{i} \in \mathrm{C}$ such that $\sum\left|a_{i}\right|^{2}=1$. Each equivalence class $[Q]$ intersects the unit sphere $\mathrm{S}$ in a circle, i.e., $[Q] \cap S=\left\{\ell Q:|\ell|=1 / \sum\left|a_{i}\right|^{2}\right\}$. We define $\operatorname{dist}([P],[Q])=\inf \left\{d\left(P_{0}, Q_{0}\right)\right.$ : $\left.P_{0} \in[P] \cap \mathrm{S}, Q_{0} \in[Q] \cap \mathrm{S}\right\}$, where $d\left(\left(a_{1}, \ldots, a_{n-1}\right),\left(b_{1}, \ldots, b_{n-1}\right)\right)=\left(\sum \mid a_{i}-\right.$ $\left.\left.b_{i}\right|^{2}\right)^{1 / 2}$ is the Euclidean distance on $S$. It is a simple exercise to verify that this defines a metric on $\hat{\mathrm{C}}$.

A $\hat{\mu}$-invariant set $A \subset \hat{C}$ is an exponential attractor with basin $B$ for $\hat{\mu}$ if there exists $c, \gamma>0$ such that $\operatorname{dist}\left(\hat{\mu}^{\mathrm{m}}([\mathrm{Q}]), \mathrm{A}\right) \leq \mathrm{c} \exp (-\gamma \mathrm{m})$ for all $m \geq 0$ and all $[Q] \in B$. If $B=\hat{C}$ then we say that $A$ is a global exponential attractor.

For each $j \in\{1, \ldots,\lceil n / 2\rceil-1\}$ let $B_{j} \subset \mathrm{C}$ be the subspace generated by $\left\{X_{j}, X_{j+1}, \ldots, X_{n-j}\right\}$. Let $B_{\lceil n / 2\rceil}:=\emptyset$, and Aff $:=\cup_{j=1}^{\lceil n / 2\rceil-1} A_{j}$. For any subset $D \subset \mathrm{C}$ let $\hat{D}:=\{[P]: P \in D\}$. We will only use this notation for sets $D$ which are maximal in the sense that if $\hat{D}=\hat{E}$ then $E \subset D$.

Theorem 5 For each $j \in\{1, \ldots,\lceil n / 2\rceil-1\}$ the set $\hat{A}_{j}$ is an exponential attractor with basin $\hat{B_{j}} \backslash \hat{B_{j+1}}$ and thus $\hat{A f f}$ is a global exponential attractor for $\hat{\mu}$. The map $\hat{\mu}$ is n-periodic (i.e., $\hat{\mu}^{n}=I d$ ) on each $\hat{A}_{j}$, and thus on $\hat{A f f}$.

Proof. Suppose $Q \in B_{j} \backslash B_{j+1}$. Equation (5) implies that the polygon $\mu^{m}(Q)=$ $\sum_{i=1}^{n-1}\left(\frac{1+q^{i}}{2}\right)^{m} a_{i} X_{i}=\sum_{i=j}^{n-j}\left(\frac{1+q^{i}}{2}\right)^{m} a_{i} X_{i}$ is $\star$-similar to

$$
\left(\frac{2}{1+q^{j}}\right)^{m} \mu^{m}(Q)=a_{j} X_{j}+\sum_{i=j+1}^{n-j-1}\left(\frac{1+q^{i}}{1+q^{j}}\right)^{m} a_{i} X_{i}+\left(\frac{1+q^{n-j}}{1+q^{j}}\right)^{m} a_{n-j} X_{n-j} .
$$

Since $\left|\left(1+q^{i}\right) /\left(1+q^{j}\right)\right|<1$ the terms in the middle sum are exponentially small. Thus $\hat{\mu}^{m}([Q])$ is exponentially close to the equivalence class $\left[P_{m}\right] \in \hat{A}_{j}$ of the polygon $P_{m}:=a_{j} X_{j}+\left[\left(1+q^{n-j}\right) /\left(1+q^{j}\right)\right]^{m} a_{n-j} X_{n-j}$. The fact that Aff is a global attractor follows from this since $\hat{C}$ is the union of the $\hat{B}_{j}$. Lemma immediately implies that $\hat{\mu}$ is periodic on each $\hat{A}_{j}$.

Acknowledgments. Many thanks to Sergei Tabachnikov and the two anonymous referees for helpful remarks. 


\section{References}

[1] J. C. Alexander, The symbolic dynamics of the sequence of pedal triangles, Math. Mag. 66 (1993) 147-158.

[2] E. R. Berlekamp, E. N. Gilbert, and F. W. Sinden, A polygon problem, this Monthly 72 (1965) 233-241.

[3] D. DeTemple and J. Robertson, A billiard path characterization of regular polygons, Math. Mag. 54 (1981) 73-75.

[4] E. Gutkin, Two applications of calculus to triangular billiards, this Monthly 104 (1997) 618-622.

[5] J. Kingston and J. Synge, The sequence of pedal triangles, this MonthLY 95 (1988) 609-620.

[6] P. Lax, The ergodic character of sequences of pedal triangles, this Monthly 97 (1990) 377-381.

[7] S. Tabachnikov, Billiards, Panoramas et Synthèses, vol. 1, Société Mathématique de France, Marseille, 1995.

[8] —, Fagnano orbits of polygonal dual billiards, Geom. Dedicata 77 (1999) 279-286.

[9] - A proof of Culter's theorem on the existence of periodic orbits in polygonal outer billiards, Geom. Dedicata 129 (2007) 83-87.

[10] S. Tabachnikov and F. Dogru, Dual billiards, Math. Intelligencer 27 (2005) 18-25.

[11] P. Ungar, Mixing property of the pedal mapping, this MonThLy 97 (1990) 898-900.

Serge E. Troubetzkoy received his B.A. from Yale University in 1982 and his Ph.D. from Stanford University in 1987. After a Brownian career path: Leningrad, Toronto, Warwick, Bielefeld, Stony Brook, and UAB, he settled down in Marseille where, due to the complicated French system, he has four affiliations (but only one salary): Centre de Physique Théorique, Fédération de Recherches des Unités de Mathématique de Marseille, Institut de Mathématiques de Luminy, and Université de la Méditerranée. His area of research is dynamical systems with emphasis on billiards. He has written articles on four types of billiards: polygonal billiards, hyperbolic billiards, elliptic billiards, and now on dual billiards.

Centre de Physique Théorique, Luminy, Case 90\%, F-13288 Marseille Cedex 9, France

troubetz@iml.univ-mrs.fr 